\title{
POLYNOMIAL MODULES OVER THE STEENROD ALGEBRA AND CONJUGATION IN THE MILNOR BASIS
}

\author{
KENNETH G. MONKS
}

(Communicated by Thomas Goodwillie)

\begin{abstract}
Let $P_{s}=\mathbb{F}_{2}\left[x_{1}, \ldots, x_{s}\right]$ be the mod 2 cohomology of the $s$ fold product of $\mathbb{R P}^{\infty}$ with the usual structure as a module over the Steenrod algebra. A monomial in $P_{S}$ is said to be hit if it is in the image of the action $\bar{A} \otimes P_{S} \rightarrow P_{S}$ where $\bar{A}$ is the augmentation ideal of $A$. We extend a result of Wood to determine a new family of hit monomials in $P_{s}$. We then use similar methods to obtain a generalization of antiautomorphism formulas of Davis and Gallant.
\end{abstract}

\section{INTRODUCTION AND MAIN RESULTS}

Let $P_{s}$ be the mod 2 cohomology of the $s$-fold product of $R P^{\infty} . P_{s}$ is isomorphic as a graded algebra to $\mathbb{F}_{2}\left[x_{1}, \ldots, x_{s}\right]$ on 1-dimensional classes $x_{i}$ and is a module over the Steenrod algebra, $A$, according to well-known rules. An element $M \in P_{s}$ is said to be hit if $M \in \bar{A} P_{s}$ where $\bar{A}$ is the augmentation ideal of $A$, i.e., if $M$ can be expressed as a finite sum of the form $\sum_{j>0} \mathrm{Sq}(j) p_{j}$ for some $p_{j} \in P_{s}$.

We are interested in the general problem of determining the family of hit monomials in $P_{s}$. The problem is an interesting and important one. Let $\alpha(n)$ be the number of ones in the binary expansion of $n$. In [W], Wood showed that if $\alpha(d+s)>s$ then every monomial of degree $d$ in $P_{s}$ is hit. This verified a conjecture of Peterson, who discusses several consequences of Wood's result in [P]. Singer later proved a generalization of Wood's result in [S], identifying a larger class of hit monomials. In [Sil], Silverman makes progress toward proving a conjecture of Singer which would identify yet another class of hit monomials. Many of the ideas and notation used in this paper were motivated by these papers.

For any integers $n \geq 0$ and $t \geq 1$ let $\gamma_{t}(n)=\sum_{i=0}^{n-1} 2^{i t}$ (take $\gamma_{t}(0)=0$ ). A sequence of nonnegative integers $K=\left(k_{1}, \ldots, k_{m}\right)$ is called a $t$-decomposition of a positive integer $n$ if $n=\sum_{i=1}^{m} \gamma_{t}\left(k_{i}\right)$. For example, a sequence of $n$ ones is a $t$-decomposition of any integer $n$. We define $\mu_{t}(n)$ to be the number of terms in the shortest $t$-decomposition of $n$, i.e., $\mu_{t}(n)=\min \left\{m \mid n=\sum_{i=1}^{m} \gamma_{t}\left(k_{i}\right)\right\}$.

Our main result is

Received by the editors September 24, 1992 and, in revised form, February 4, 1993.

1991 Mathematics Subject Classification. Primary 55S10, 55S05; Secondary $20 \mathrm{~J} 05$. 
Theorem 1.1. Suppose $M$ is a monomial in $P_{s}$ of the form $M=E F^{2^{t}}$ where $E$ and $F$ are monomials of degree $e$ and $f$ respectively. If $e<\mu_{t}(f)$ then $M$ is hit.

This generalizes a result of Wood [W], who proved Theorem 1.1 for $t=1$. Singer proved a generalization of Wood's result, and we refer the reader to [S] for a precise statement of his result. Theorem 1.1 identifies a new class of hit monomials not determined by these previous results. For example, applying Theorem 1.1 with $t=3$ shows that the monomial $x_{1} x_{2} x_{3} x_{4} x_{5} x_{6} x_{7} x_{8}^{32} x_{9}^{32} x_{10}^{32} x_{11}^{32}$ is hit, a fact which does not follow from either of the previous theorems mentioned. Our result does not generalize Singer's result, however, as his theorem shows, for example, that $x_{1} x_{2}^{5}$ is hit, while this cannot be deduced from Theorem 1.1.

Silverman [Sil] discusses a conjecture attributed to Singer which states that Theorem 1.1 would still be true if we replaced the condition $e<\mu_{t}(f)$ by the condition $e<\left(2^{t}-1\right) \mu_{1}(f)$. We will show that $\mu_{t}(f) \leq\left(2^{t}-1\right) \mu_{1}(f)$ for all $t, f \geq 1$. Thus Theorem 1.1 proves Singer's conjecture for all $t, f$ such that $\mu_{t}(f)=\left(2^{t}-1\right) \mu_{1}(f)$.

We should also note that $\mu_{t}(f)$ is quite easy to compute, a fact which may not be obvious from the definition. In fact, we can define a recursive function from the positive integers to the set of finite sequences of positive integers by

$$
f_{t}(n)= \begin{cases}p & \text { if } n=\gamma_{t}(p), \\ p, f_{t}\left(n-\gamma_{t}(p)\right) & \text { if } \gamma_{t}(p)<n<\gamma_{t}(p+1) .\end{cases}
$$

It easily follows from [Gal, Proposition 2] that $f_{t}(n)$ is a shortest $t$-decomposition of $n$. For example, $f_{2}(59)=3,3,2,2,2,1,1$, so $\mu_{2}(59)=7$. Hence Theorem 1.1 is quite easy to use in practice.

Let $\mathrm{Sq}_{t}\left(r_{1}, \ldots, r_{m}\right)$ be the Milnor basis element $\mathrm{Sq}\left(s_{1}, \ldots, s_{t m}\right)$ where $s_{t i}=r_{i}$ and $s_{j}=0$ if $t$ does not divide $j$. For example, $\mathrm{Sq}_{3}(2,3)=$ $\mathrm{Sq}(0,0,2,0,0,3)$. For a given $t$ the set of all such elements forms a basis for a Hopf subalgebra of $A$ which we will call $B_{t}$. The essential idea in our proof of Theorem 1.1 is that it is often easier to show that a given monomial is hit by working in one of these subalgebras rather than the entire Steenrod algebra. By studying the number-theoretic properties of the excesses of elements in these subalgebras, we can also prove the following result. Let $\chi$ be the canonical antiautomorphism of the Steenrod algebra.

Theorem 1.2. For any integers $s, t$, and $k$ such that $s, t \geq 1,0 \leq k<s$, and $k \leq t$ we have

$$
\chi \mathrm{Sq}_{t}\left(2^{s}-2^{k}\right)=\mathrm{Sq}_{t}\left(2^{s-1}\right) \mathrm{Sq}_{t}\left(2^{s-2}\right) \cdots \mathrm{Sq}_{t}\left(2^{k}\right) .
$$

This generalizes results of Davis [D], who proved Theorem 1.2 for the case $t=1$, and of Gallant [Gal], who proved Theorem 1.2 for $k=0$ and all $t$. Both Gallant's proof and our own imitate Davis's original proof closely.

\section{PRoperties OF THE MiNimuM EXCESS FUNCTION}

Recall that the excess of any element $\theta \in A$ is given by

$$
\operatorname{ex}(\theta)=\min \left\{s \mid \theta\left(x_{1} x_{2} \cdots x_{s}\right) \neq 0 \in P_{s}\right\}
$$


and that, for any $u \in P_{s}$ of degree $k, \theta u=0$ if $\operatorname{ex}(\theta)>k$. It is shown in $[\mathrm{K}]$ that the excess of Milnor basis elements can easily be computed by $\operatorname{ex}\left(\operatorname{Sq}\left(r_{1}, \ldots, r_{m}\right)\right)=\sum_{i=1}^{m} r_{i}$ and that, for an arbitrary $\theta \in A, \operatorname{ex}(\theta)$ is the minimum of the excesses of the Milnor basis summands of $\theta$.

For any finite sequence $R=\left(r_{1}, \ldots, r_{m}\right)$ of nonnegative integers let $\operatorname{Sq}_{t}(R)$ denote the Milnor basis element $\mathrm{Sq}_{t}\left(r_{1}, \ldots, r_{m}\right)$. The dimension of an arbitrary Milnor basis element $\operatorname{Sq}\left(r_{1}, \ldots, r_{m}\right)$ is $\sum_{i=1}^{m}\left(2^{i}-1\right) r_{i}$. Consequently, the dimension of $\mathrm{Sq}_{t}\left(r_{1}, \ldots, r_{m}\right)$ is $\sum_{i=1}^{m}\left(2^{t i}-1\right) r_{i}$. As in the introduction, for a fixed $t$ let $B_{t}$ be the vector subspace of $A$ with basis the set of all $\mathrm{Sq}_{t}(R)$. One can easily verify [Gal], either directly or by using the results in [AD], that $B_{t}$ is a Hopf subalgebra of $A$. We note that $B_{1}=A$ and that all of the nonzero elements of $B_{t}$ are in dimensions divisible by $\left(2^{t}-1\right)$.

Notice that for any $t$ and $k$

$$
\left(2^{t}-1\right) \gamma_{t}(k)=\left(2^{t k}-1\right) \text {. }
$$

Consequently, $n=\sum_{i=1}^{m} \gamma_{t}\left(k_{i}\right)$ if and only if $\left(2^{t}-1\right) n=\sum_{i=1}^{m}\left(2^{t k_{i}}-1\right)$.

Let $\mathrm{Sq}_{t}\left(r_{1}, \ldots, r_{m}\right)$ be any Milnor basis element in dimension $\left(2^{t}-1\right) n$. Then by the dimension formula

$$
\left(2^{t}-1\right) n=\sum_{i=1}^{m}\left(2^{t i}-1\right) r_{i}=\left(2^{t}-1\right) \sum_{i=1}^{m} \sum_{j=1}^{r_{i}} \gamma_{t}(i)
$$

so

$$
n=\sum_{i=1}^{m} \sum_{j=1}^{r_{i}} \gamma_{t}(i)
$$

This expression provides us with a $t$-decomposition of $n$ of length $\sum_{i=1}^{m} r_{i}$, the excess of $\operatorname{Sq}_{t}\left(r_{1}, \ldots, r_{m}\right)$. It is elementary to check that this correspondence between Milnor basis elements $\theta$ in $B_{t}$ in dimension $\left(2^{t}-1\right) n$ and $t$-decompositions of $n$ of length $\operatorname{ex}(\theta)$ is one-to-one (modulo reordering of the $t$-decompositions and interspersion of zeros). Thus the value of $\mu_{t}(n)$ is exactly the excess of the element of $B_{t}$ in dimension $\left(2^{t}-1\right) n$ having least excess, i.e.,

$$
\mu_{t}(n)=\min \left\{\operatorname{ex}(\theta) \mid \theta \in B_{t}, \operatorname{dim}(\theta)=\left(2^{t}-1\right) n\right\} .
$$

Accordingly, we will call $\mu_{t}$ the minimum excess function (for the subalgebra $B_{t}$ ). In particular, all elements of $A$ in dimension $n$ have excess greater than or equal to $\mu_{1}(n)$.

We now establish some number-theoretic properties of the minimum excess function that will be needed for our proofs of the main results. We begin by proving the fact referred to in the introduction.

Lemma 2.1. $\mu_{t}(n) \leq\left(2^{t}-1\right) \mu_{1}(n)$ for all $t, n \geq 1$.

Proof. By the definition of $\mu_{1}(n)$ there are positive integers $k_{1}, k_{2}, \ldots, k_{\mu_{1}(n)}$ such that

$$
n=\sum_{i=1}^{\mu_{1}(n)} \gamma_{1}\left(k_{i}\right)
$$


For each $k_{i}$ let $k_{i}=t q_{i}+r_{i}$ where $q_{i}$ and $r_{i}$ are nonnegative integers and $0 \leq r_{i}<t$. Then using (2.2) we have

$$
\begin{aligned}
n & =\sum_{i=1}^{\mu_{1}(n)} \gamma_{1}\left(t q_{i}+r_{i}\right)=\sum_{i=1}^{\mu_{1}(n)}\left(2^{t q_{i}+r_{i}}-1\right) \\
& =\sum_{i=1}^{\mu_{1}(n)}\left[\left(2^{t}-2^{r_{i}}\right) \frac{\left(2^{t q_{i}}-1\right)}{\left(2^{t}-1\right)}+\left(2^{r_{i}}-1\right) \frac{\left(2^{t\left(q_{i}+1\right)}-1\right)}{\left(2^{t}-1\right)}\right] \\
& =\sum_{i=1}^{\mu_{1}(n)}\left[\sum_{j=1}^{2^{t}-2^{r_{i}}} \gamma_{t}\left(q_{i}\right)+\sum_{j=1}^{2^{r_{i}}-1} \gamma_{t}\left(q_{i}+1\right)\right],
\end{aligned}
$$

the last expression yielding a $t$-decomposition of $n$ having at most $\left(2^{t}-1\right) \mu_{1}(n)$ terms. Hence, $\mu_{t}(n) \leq\left(2^{t}-1\right) \mu_{1}(n)$.

We will require more information about $\mu_{t}$.

Lemma 2.2. $\mu_{t}(n)=n$ if $n \leq 2^{t}$.

Proof. Since $n \leq 2^{t}$, we have $n \leq 2^{t}<2^{t}+1=\gamma_{t}(2)$. Since $\gamma_{t}$ is strictly increasing, the only possible $t$-decomposition of $n$ is a sequence of $n$ ones.

We now need some new notation. Let $K=\left(k_{1}, \ldots, k_{m}\right)$ be any sequence of nonnegative integers. Define

$$
\begin{gathered}
|K|=\sum_{i=1}^{m} k_{i}, \\
\nu(K)=\max _{i}\left\{k_{i}\right\},
\end{gathered}
$$

and

$$
V_{t}(K)=\sum_{i=1}^{m} \gamma_{t}\left(k_{i}\right)
$$

Thus every such sequence $K$ is a $t$-decomposition of $V_{t}(K)$.

Now assume that $k_{1} \geq k_{2} \geq \cdots \geq k_{m}$ (so that $\nu(K)=k_{1}$ ) and also that $|K| \geq 1$. For such a sequence define

$$
\delta(K)=\left(k_{1}^{\prime}, \ldots, k_{m}^{\prime}\right)
$$

by

$$
k_{i}^{\prime}= \begin{cases}k_{i}-1 & \text { if } k_{i}=k_{1} \text { and }\left(k_{i+1} \neq k_{1} \text { or } i=m\right) \\ k_{i} & \text { otherwise }\end{cases}
$$

It is easy to see that

$$
\begin{gathered}
k_{1}^{\prime} \geq k_{2}^{\prime} \geq \cdots \geq k_{m}^{\prime}, \\
|\delta(K)|=|K|-1, \\
\nu(\delta(K)) \leq \nu(K),
\end{gathered}
$$


and

$$
V_{t}(\delta(K))=V_{t}(K)-2^{t(\nu(K)-1)} .
$$

By (2.13) and (2.14) we can define $\delta^{r}$ for $0 \leq r \leq|K|$ to be the $r$ fold composition of $\delta$ with itself $\left(\delta^{0}=\right.$ the identity function). Let $D_{K}=$ $\left(d_{1}, d_{2}, \ldots, d_{|K|}\right)$ be the sequence given by

$$
d_{i}=V_{t}\left(\delta^{i-1}(K)\right)-V_{t}\left(\delta^{i}(K)\right) \text {. }
$$

Then by (2.16) we have $d_{i}=2^{t\left(\nu\left(\delta^{i-1}(K)\right)-1\right)}$, so by (2.15) we have $d_{1} \geq d_{2} \geq$ $\cdots \geq d_{|K|}$. Noting that $\delta^{|K|}(K)=(0, \ldots, 0)$ we see that $V_{t}\left(\delta^{|K|}(K)\right)=0$, so

$$
\begin{aligned}
\left|D_{K}\right| & =\sum_{i=1}^{|K|}\left[V_{t}\left(\delta^{i-1}(K)\right)-V_{t}\left(\delta^{i}(K)\right)\right] \\
& =V_{t}\left(\delta^{0}(K)\right)-V_{t}\left(\delta^{|K|}(K)\right)=V_{t}(K) .
\end{aligned}
$$

Armed with this notation we are ready to prove

Lemma 2.3. If $n<2^{s}$ then $\mu_{t}(n) \leq \mu_{t}\left(n+2^{s}\right)$.

Proof. It suffices to show that for any $t$-decomposition of $n+2^{s}$ of length $m$ there is a $t$-decomposition of $n$ of length at most $m$. So assume that $K=\left(k_{1}, \ldots, k_{m}\right)$ is a $t$-decomposition of $n+2^{s}$. Without loss of generality we may further assume that $k_{1} \geq k_{2} \geq \cdots \geq k_{m}$. By definition we have $V_{t}(K)=n+2^{s}$, so by $(2.18)$

$$
\sum_{i=0}^{|K|} d_{i}=n+2^{s}
$$

Thus $D_{K}$ is a nonincreasing sequence of two powers whose sum is $n+2^{s}$. Notice that $n<2^{s}$ implies that $2^{s}<n+2^{s}<2^{s+1}$. This allows us to make use of the following

Lemma 2.4. Let $a$ be any positive integer, and let $2^{b}$ be the largest two power less than or equal to $a$. Then any nonincreasing sequence of two powers whose sum is $a$ has an initial subsequence whose sum is $2^{b}$.

In other words, if $2^{b} \leq a<2^{b+1}, \sum_{i=1}^{r} 2^{p_{i}}=a$, and $2^{p_{1}} \geq \cdots \geq 2^{p_{r}}$, then there exists $q \in\{1, \ldots, r\}$ such that $\sum_{i=1}^{q} 2^{p_{i}}=2^{b}$.

Proof (of Lemma 2.4). If $a=2^{b}$, we can take $q=r$ and we are finished. Assume $a>2^{b}$. Since $2^{b+1}>a$, we must have $2^{b} \geq 2^{p_{1}} \geq \cdots \geq 2^{p_{q+1}}$. Let $q$ be the largest integer such that $\sum_{i=1}^{q} 2^{p_{i}} \leq 2^{b}$. Then $2^{b}-\sum_{i=1}^{q} 2^{p_{i}} \equiv 0$ $\bmod 2^{p_{q+1}}$ and $2^{b}<\sum_{i=1}^{q+1} 2^{p_{i}}$, so $\sum_{i=1}^{q} 2^{p_{i}}=2^{b}$.

Applying this result to the case at hand we see that there must be an integer $q \in\{1, \ldots,|K|\}$ such that $\sum_{i=1}^{q} d_{i}=2^{s}$. Thus

$$
\begin{aligned}
\sum_{i=0}^{q} V_{t}\left(\delta^{i-1}(K)\right)-V_{t}\left(\delta^{i}(K)\right) & =V_{t}(K)-V_{t}\left(\delta^{q}(K)\right) \\
& =n+2^{s}-V_{t}\left(\delta^{q}(K)\right)=2^{s},
\end{aligned}
$$


so $V_{t}\left(\delta^{q}(K)\right)=n$. Thus $\delta^{q}(K)$ is a $t$-decomposition of $n$ of length $m$, so $\mu_{t}(n) \leq \mu_{t}\left(n+2^{s}\right)$.

Using this result we can now prove

Lemma 2.5. If $s, t$, and $k$ are any integers such that $s, t \geq 1,0 \leq k<s$, and $k \leq t$ then $\mu_{t}\left(2^{s}-2^{k}\right) \geq 2^{k}$.

Proof. We will proceed by induction on $s$. If $s=k+1$ then $\mu_{t}\left(2^{s}-2^{k}\right)=$ $\mu_{t}\left(2^{k}\right)=2^{k}$ by Lemma 2.2. Assume the lemma is true for $s-1$. Then

$$
\mu_{t}\left(2^{s}-2^{k}\right)=\mu_{t}\left(2^{s-1}+\left(2^{s-1}-2^{k}\right)\right) \geq \mu_{t}\left(2^{s-1}-2^{k}\right) \geq 2^{k}
$$

where the first inequality follows from Lemma 2.3 and the second from our inductive hypothesis.

\section{Hit elements in $P_{s}$}

In this section we will prove Theorem 1.1 by combining the key idea from [W] with the results of the last section and some facts about the antiautomorphism in the Milnor basis.

The key idea in Wood's argument is that for any $u, w \in P_{s}$ and any $\theta \in A$ we have $u \cdot \theta w \equiv \chi \theta u \cdot w$ modulo hit elements. Thus if $\chi \theta u=0$ or in particular if $\operatorname{ex}(\chi \theta)>\operatorname{deg}(u)$ then $u \cdot \theta w$ is hit. As in the theorem, suppose $M$ is a monomial in $P_{s}$ of the form $M=E F^{2^{t}}$ where $E$ and $F$ are monomials of degree $e$ and $f$ respectively. In order to use Wood's argument in this setting, we would like to express $M$ in the form $u \cdot \theta w$ for some $u, w \in P_{s}$ and some $\theta \in A$. This is accomplished with the aid of the following lemma.

Lemma 3.1. Let $M \in P_{s}$ be any element of degree $k$. Then for any $t \geq 1$

$$
\mathrm{Sq}_{t}(k) M=M^{2^{t}} \text {. }
$$

Proof. As exponentiation by a power of 2 distributes across sums in $P_{s}$, it suffices to prove the result when $M$ is a monomial. Recall that $A^{*}$, the Hopf dual of $A$, is isomorphic to the graded polynomial algebra $F_{2}\left[\xi_{1}, \xi_{2}, \ldots\right]$ where $\xi_{t}$ is in dimension $2^{t}-1$ [Mil]. The Milnor basis of $A$ is dual to the basis of monomials in $A^{*}$. Let $\xi=\sum_{i=0}^{\infty} \xi_{i}$. If $\theta \in A$ is an element of degree $n$ then $\theta x^{k}=\left\langle\theta, \xi^{k}\right\rangle x^{n+k}$ for any 1 -dimensional class $x \in P_{s}$ [BDP]. Thus in particular

$$
\mathrm{Sq}_{t}(k) x^{k}=\left\langle\mathrm{Sq}_{t}(k), \xi^{k}\right\rangle x^{\left(2^{t}-1\right) k+k}=\left(x^{k}\right)^{2^{t}}
$$

since $\xi_{t}^{k}$ is a summand of $\xi^{k}$.

We now proceed by induction on the number of variables in $M$. Suppose $M=x_{i_{1}}^{e_{1}} x_{i_{2}}^{e_{2}} \cdots x_{i_{m}}^{e_{m}}$. If $m=1$, the lemma is true by (3.1). Assume the lemma is true for all monomials comprised of less than $m$ variables. Let $M_{1}=x_{i_{1}}^{e_{1}} x_{i_{2}}^{e_{2}} \cdots x_{i_{m-1}}^{e_{m-1}}$ so that $M=M_{1} x_{i_{m}}^{e_{m}}$. Let $\Delta: A \rightarrow A \otimes A$ be the diagonal map of $A$. Then $\Delta \mathrm{Sq}_{t}(k)=\sum_{i=0}^{k} \mathrm{Sq}_{t}(k-i) \otimes \mathrm{Sq}_{t}(i)$ [Mil]. Noting that $\sum_{i=0}^{e_{m}-1} \mathrm{Sq}_{t}(k-i) M_{1} \cdot \operatorname{Sq}_{t}(i) x_{i_{m}}^{e_{m}}=0$ because $\operatorname{ex}\left(\operatorname{Sq}_{t}(k-i)\right)>\operatorname{deg}\left(M_{1}\right)$ and that 
$\sum_{i=e_{m}+1}^{k} \mathrm{Sq}_{t}(k-i) M_{1} \cdot \mathrm{Sq}_{t}(i) x_{i_{m}}^{e_{m}}=0$ because $\operatorname{ex}\left(\mathrm{Sq}_{t}(i)\right)>\operatorname{deg}\left(x_{i_{m}}^{e_{m}}\right)$, we obtain

$$
\begin{aligned}
\mathrm{Sq}_{t}(k) \cdot M= & \sum_{i=0}^{k} \mathrm{Sq}_{t}(k-i) M_{1} \cdot \mathrm{Sq}_{t}(i) x_{i_{m}}^{e_{m}} \\
= & \sum_{i=0}^{e_{m}-1} \mathrm{Sq}_{t}(k-i) M_{1} \cdot \mathrm{Sq}_{t}(i) x_{i_{m}}^{e_{m}} \\
& +\mathrm{Sq}_{t}\left(k-e_{m}\right) M_{1} \cdot \mathrm{Sq}_{t}\left(e_{m}\right) x_{i_{m}}^{e_{m}} \\
& +\sum_{i=e_{m}+1}^{k} \mathrm{Sq}_{t}(k-i) M_{1} \cdot \mathrm{Sq}_{t}(i) x_{i_{m}}^{e_{m}} \\
= & \operatorname{Sq}_{t}\left(k-e_{m}\right) M_{1} \cdot \mathrm{Sq}_{t}\left(e_{m}\right) x_{i_{m}}^{e_{m}} \stackrel{\text { ind }}{=} M_{1}^{2^{t}}\left(x_{i_{m}}^{e_{m}}\right)^{2^{t}}=M^{2^{t}} .
\end{aligned}
$$

Using this we can express $M$ in the desired form

$$
M=E \cdot F^{2^{t}}=E \cdot \mathrm{Sq}_{t}(f) F .
$$

Applying Wood's argument shows that $M \equiv \chi \mathrm{Sq}_{t}(f) E \cdot F$ modulo hit elements. Thus if $\operatorname{ex}\left(\chi \operatorname{Sq}_{t}(f)\right)>e$ then $\chi \operatorname{Sq}_{t}(f) E=0$, and consequently $M$ is hit. Thus to prove the theorem it suffices to show that $\operatorname{ex}\left(\chi \mathrm{Sq}_{t}(f)\right)=\mu_{t}(f)$.

Lemma 3.2. $\chi \mathrm{Sq}_{t}(n)$ is the sum of all $\mathrm{Sq}_{t}(R)$ in dimension $\left(2^{t}-1\right) n$.

Lemma 3.2 follows directly from the formula for $\chi$ of a Milnor basis element [Mil, Theorem 5] as in [Gal, Proposition 1] and was proved by completely different means by the author [M].

With this at our disposal, all that remains is to recall from (2.5) that $\mu_{t}(f)$ is precisely the excess of the element $\operatorname{Sq}_{t}(R)$ in dimension $\left(2^{t}-1\right) f$ having minimal excess. As every $\operatorname{Sq}_{t}(R)$ in dimension $\left(2^{t}-1\right) f$ is a summand of $\chi \mathrm{Sq}_{t}(f), \operatorname{ex}\left(\chi \mathrm{Sq}_{t}(f)\right)=\mu_{t}(f)$, completing the proof of Theorem 1.1.

\section{APPLICATION TO THE ANTIAUTOMORPHISM}

In this section we will use the properties of the minimum excess function developed above to prove Theorem 1.2. We imitate closely the original method used by Davis in [D] in which he proves Theorem 1.2 for the case $t=1$ and which was used in [Gal] in which he proves Theorem 1.2 for the case $k=0$ and all $t$. Accordingly we begin by stating

Proposition 4.1 [Gal, Corollary 1(a)].

$$
\mathrm{Sq}_{t}(m) \chi \mathrm{Sq}_{t}(l)=\sum\left(\begin{array}{c}
\sum 2^{t(i-1)} r_{i} \\
m
\end{array}\right) \mathrm{Sq}_{t}(R)
$$

where the summation is taken over all $\mathrm{Sq}_{t}(R)\left(=\operatorname{Sq}_{t}\left(r_{1}, r_{2}, \ldots\right)\right)$ in dimension $\left(2^{t}-1\right)(m+l)$.

Using this and our previous results we are now ready to prove

Theorem 1.2. For any integers $s, t$, and $k$ such that $s, t \geq 1,0 \leq k<s$, and $k \leq t$ we have

$$
\chi \mathrm{Sq}_{t}\left(2^{s}-2^{k}\right)=\mathrm{Sq}_{t}\left(2^{s-1}\right) \mathrm{Sq}_{t}\left(2^{s-2}\right) \cdots \mathrm{Sq}_{t}\left(2^{k}\right)
$$


Proof. We proceed by induction on $s$ (for a given $k$ and $t$ ). Suppose $s=k+1$. It is easily checked that for $k \leq t$ the only nonzero element of $B_{t}$ in dimension $\left(2^{t}-1\right) 2^{k}$ is $\operatorname{Sq}_{t}\left(2^{k}\right)$. Thus $\chi \mathrm{Sq}_{t}\left(2^{s}-2^{k}\right)=\chi \mathrm{Sq}_{t}\left(2^{k}\right)=\mathrm{Sq}_{t}\left(2^{k}\right)$, proving the theorem for $s=k+1$.

Now assume the theorem holds for $s-1$. By Proposition 4.1 and the wellknown fact that

$$
\left(\begin{array}{l}
x \\
y
\end{array}\right) \equiv\left(\begin{array}{l}
2^{w} x \\
2^{w} y
\end{array}\right) \quad \bmod 2
$$

we obtain

$$
\begin{gathered}
\mathrm{Sq}_{t}\left(2^{s-1}\right) \mathrm{Sq}_{t}\left(2^{s-2}\right) \cdots \mathrm{Sq}_{t}\left(2^{k}\right) \stackrel{\text { ind }}{=} \mathrm{Sq}_{t}\left(2^{s-1}\right) \chi \mathrm{Sq}_{t}\left(2^{s-1}-2^{k}\right) \\
=\sum_{R}\left(\begin{array}{c}
\sum 2^{t(i-1)} r_{i} \\
2^{s-1}
\end{array}\right) \mathrm{Sq}_{t}(R)=\sum_{R}\left(\begin{array}{c}
\sum 2^{t i} r_{i} \\
2^{s+t-1}
\end{array}\right) \mathrm{Sq}_{t}(R)
\end{gathered}
$$

where the sums on the right-hand side are taken over all sequences $R=$ $\left(r_{1}, \ldots, r_{m}\right)$ such that $\mathrm{Sq}_{t}(R)$ is in dimension $\left(2^{t}-1\right)\left(2^{s}-2^{k}\right)$. By Lemma $3.2 \chi \mathrm{Sq}_{t}\left(2^{s}-2^{k}\right)$ is the sum of all $\mathrm{Sq}_{t}(R)$ in dimension $\left(2^{t}-1\right)\left(2^{s}-2^{k}\right)$. So it suffices to show that $\left(\sum_{2^{s+t-1}}^{2^{i t} r_{i}}\right)$ is odd in every summand of the last expression of (4.2). There is an old well-known theorem of Lucas [L] that says $\left(\begin{array}{l}x \\ y\end{array}\right)$ is odd if and only if $\prod_{i}\left(\begin{array}{c}\alpha_{i}(x) \\ \alpha_{i}(y)\end{array}\right)$ is odd where $x=\sum_{i} \alpha_{i}(x) 2^{i}$ and $y=\sum_{i} \alpha_{i}(y) 2^{i}$ are the binary expansions of $x$ and $y$. Thus it suffices to show that

$$
2^{s+t-1} \leq \sum 2^{t i} r_{i}<2^{s+t}
$$

for every $R$ in the last expression of (4.2), which we now prove.

Since $\mathrm{Sq}_{t}(R)$ is in dimension $\left(2^{t}-1\right)\left(2^{s}-2^{k}\right)$, we have

$$
\sum_{i}\left(2^{t i}-1\right) r_{i}=\left(2^{t}-1\right)\left(2^{s}-2^{k}\right),
$$

so

$$
\sum_{i} 2^{t i} r_{i}=\sum_{i} r_{i}+\left(2^{t}-1\right)\left(2^{s}-2^{k}\right)
$$

Clearly

$$
\left(2^{t}-1\right) \sum_{i} r_{i} \leq \sum_{i}\left(2^{t i}-1\right) r_{i}
$$

which together with (4.4) implies

$$
\sum_{i} r_{i} \leq\left(2^{s}-2^{k}\right) .
$$

Combining (4.5) and (4.7) yields

$$
\begin{aligned}
\sum_{i} 2^{t i} r_{i} & =\sum_{i} r_{i}+\left(2^{t}-1\right)\left(2^{s}-2^{k}\right) \\
& \leq\left(2^{s}-2^{k}\right)+\left(2^{t}-1\right)\left(2^{s}-2^{k}\right)=2^{s+t}-2^{k+t}<2^{s+t},
\end{aligned}
$$

proving the right-hand inequality of (4.3). 
To prove the other inequality we note that by $(2.5)$ we must have

$$
\sum_{i} r_{i} \geq \mu_{t}\left(2^{s}-2^{k}\right)
$$

But by Lemma $2.5 \mu_{t}\left(2^{s}-2^{k}\right) \geq 2^{k}$ so that

$$
\sum_{i} r_{i} \geq 2^{k}
$$

Now since $s>k$ and $t \geq 1$, we have

$$
\left(2^{t}-1\right)\left(2^{s}-2^{k}\right)+2^{k}-2^{s+t-1}=\left(2^{s}-2^{k+1}\right)\left(2^{t-1}-1\right) \geq 0,
$$

so it follows that

$$
2^{k}+\left(2^{t}-1\right)\left(2^{s}-2^{k}\right) \geq 2^{s+t-1} .
$$

Combining (4.5), (4.10), and (4.12) yields

$$
\begin{aligned}
\sum_{i} 2^{t i} r_{i} & =\sum_{i} r_{i}+\left(2^{t}-1\right)\left(2^{s}-2^{k}\right) \\
& \geq 2^{k}+\left(2^{t}-1\right)\left(2^{s}-2^{k}\right) \geq 2^{s+t-1}
\end{aligned}
$$

which proves the left-hand inequality of $(4.3)$ and completes the proof.

\section{ACKNOWLEDGMENT}

I would like to thank Judith Silverman and Don Davis for their many helpful discussions and comments during the course of this work.

\section{REFERENCES}

[AD] D. W. Anderson and D. M. Davis, $A$ vanishing theorem in homological algebra, Comment. Math. Helv. 48 (1973), 318-327.

[BDP] E. H. Brown, D. M. Davis, and F. P. Peterson, The homology of $B O$ and some results about the Steenrod algebra, Math. Proc. Cambridge Philos. Soc. 81 (1977), 393-398.

[D] D. M. Davis, The antiautomorphism of the Steenrod algebra, Proc. Amer. Math. Soc. 44 (1974), 235-236.

[Gal] A. M. Gallant, Excess and conjugation in the Steenrod algebra, Proc. Amer. Math. Soc. 76 (1979), 161-166.

[K] D. Kraines, On excess in the Milnor basis, Bull. London Math. Soc. 3 (1971), 363-365.

[L] E. Lucas, Theorie des functions numériques simplement periodiques, Amer. J. Math. 1 (1878), 184-240, 289-321.

[Mil] J. Milnor, The Steenrod algebra and its dual, Ann. of Math. (2) 67 (1958), 150-171.

[M] K. G. Monks, Nilpotence and torsion in the Steenrod algebra and its cohomology, Ph.D. Thesis, Lehigh Univ., 1989.

[P] F. P. Peterson, A-generators for certain polynomial algebras, Math. Proc. Cambridge Philos. Soc. 105 (1989), 311-312.

[Sil] J. H. Silverman, Conjugation and excess in the Steenrod algebra, Proc. Amer. Math. Soc. 119 (1993), 657-661. 
[S] W. Singer, On the action of Steenrod squares on polynomial algebras, Proc. Amer. Math. Soc. 111 (1991), 577-583.

[W] R. M. W. Wood, Steenrod squares of polynomials and the Peterson conjecture, Math. Proc. Cambridge Philos. Soc. 105 (1989), 307-309.

Department of Mathematics, University of Scranton, Scranton, Pennsylvania 18510

$E$-mail address: monksejaguar.uofs .edu 